\title{
Microscopic origin of training in exchange bias system
}

Amitesh Paul, and Stefan Mattauch

Citation: Appl. Phys. Lett. 95, 092502 (2009);

View online: https://doi.org/10.1063/1.3211857

View Table of Contents: http://aip.scitation.org/toc/apl/95/9

Published by the American Institute of Physics

\section{Articles you may be interested in}

Manipulation of uncompensated moments in trained exchange bias system

Applied Physics Letters 97, 032505 (2010); 10.1063/1.3457440

Exchange Anisotropy-A Review

Journal of Applied Physics 33, 1328 (2004); 10.1063/1.1728716

Simple model for thin ferromagnetic films exchange coupled to an antiferromagnetic substrate Journal of Applied Physics 62, 3047 (1998); 10.1063/1.339367

Surface-anisotropy and training effects of exchange bias in nanoparticles with inverted ferromagneticantiferromagnetic core-shell morphology

Journal of Applied Physics 110, 033908 (2011); 10.1063/1.3611417

Exchange bias training relaxation in spin glass/ferromagnet bilayers

Applied Physics Letters 108, 172401 (2016); 10.1063/1.4947287

Investigation on the origin of exchange bias in epitaxial, oriented and polycrystalline $\mathrm{Fe}_{3} \mathrm{O}_{4}$ thin films

AIP Advances 5, 117123 (2015); 10.1063/1.4935787

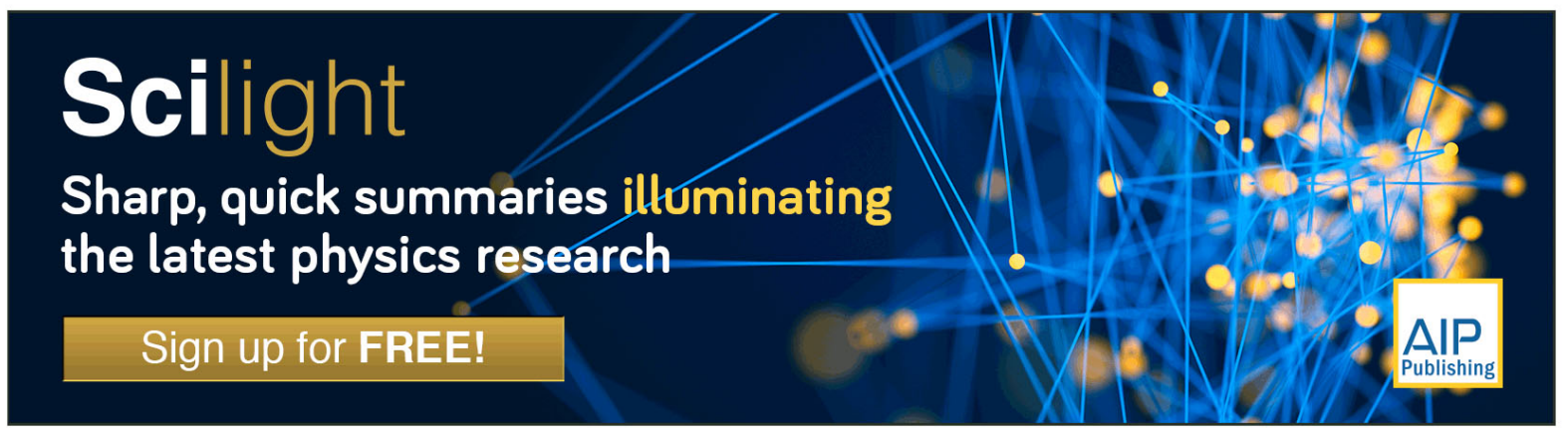




\title{
Microscopic origin of training in exchange bias system
}

\author{
Amitesh Paul ${ }^{1, \text { a) }}$ and Stefan Mattauch ${ }^{2}$ \\ ${ }^{1}$ Helmholtz-Zentrum Berlin für Materialien und Energie GmbH, Glienicker Str. 100, \\ D-14109 Berlin, Germany \\ ${ }^{2}$ Institut für Festkörperforschung IFF-4 “Streumethoden," JCNS Forschungszentrum Jülich GmbH, \\ D-52425 Jülich, Germany
}

(Received 14 July 2009; accepted 1 August 2009; published online 2 September 2009)

\begin{abstract}
The microscopic origin of training in exchange coupled systems has been identified from our experimentally observed microscopic suppression of training. It is an interplay of uniaxial anisotropy and uncompensated spins in the antiferromagnet grains that are rotatable in polycrystalline antiferromagnetic layer similar to spin-glass-like behavior. (C) 2009 American Institute of Physics. [DOI: 10.1063/1.3211857]
\end{abstract}

Interfacial exchange coupling between a ferromagnet (FM) and an antiferromagnet (AF) can "lock" the magnetization into the FM in a well-defined direction. This effect, which in a phenomenological picture takes the form of a unidirectional magnetic anisotropy is known as exchange bias. ${ }^{1}$ Exchange biasing has a significant technological importance in magnetic sensorics and high-density magnetic data storage, which demands a complete under standing of various other related phenomena, such as coercivity enhancement, asymmetric hysteresis loops, and training effect. ${ }^{2,3}$

Within the domain state model, ${ }^{4}$ the exchange field is provided by the remanent magnetization of domains in the AF during different field cooling procedures. This remanent magnetization is influenced by the magnetization state of the FM, i.e., if the FM is divided into almost equal proportion of domains (multidomain) or it is already in a saturated state (single domain). ${ }^{5}$ Thus a cooling field $H_{\mathrm{FC}}$ (cooling below the ordering temperature of $\mathrm{AF}$ ) essentially determines the state of the FM which in-turn determines the strength of the bias field $H_{E B}{ }^{6,7}$ Difference between subsequent (partial) magnetization reversal loops is called the "training effect." Even though the microscopic origin of the training effect is still under debate, it is generally agreed to be due to rearrangement of interfacial AF spin structure, which can be considered as rotatable hysteretic grains (rotatable anisotropy) particularly in polycrystalline specimens. ${ }^{5,8}$

In pursuit of understanding the origin of training effect, there have been attempts in regaining the untrained state but mainly by manipulating the field (external) strengths or the field direction. ${ }^{8}$ However, such attempts have been seen to modify the interface magnetization from the field cooled state. $^{9}$ Following superconducting quantum interference devise (SQUID) measurements, Paul et al. ${ }^{5}$ have shown that cooling the system in a remanent state (rendering remanent exchange bias), may even lead to a macroscopic suppression of the training effect, whereas the same sample being fieldcooled exhibits a clear training response. This was surprising as well as interesting as neither the temperature nor the field has been modified in regaining the untrained state. Suppression of training is not trivial either. It would have been trivial if the system has been cooled from a demagnetized state of the FM (the direction of FM domains that can couple the AF

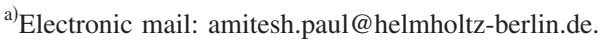

moments being randomly oriented, would have obviously resulted in no $\left.H_{E B}\right)$. Interestingly, the system here is cooled in a remanent state and posses a large remanent $H_{E B}$ and is therefore expected to behave as a normal (as the FM is magnetized in a certain direction) field cooled system.

SQUID results are averaged over a macroscopic sample (usually measuring the longitudinal component only) and cannot provide details of the micromagnetic mechanism involving the interfacial state and the related domains within the system. On the other hand, depth sensitive neutron scattering under grazing incidence with polarization analysis (PNR) has been proven decisive for the identification of reversal mechanisms in exchange bias systems. ${ }^{6,10}$ Due to the interaction between polarized neutrons and magnetic moments in the specimen, PNR is sensitive to the in-plane magnetization for a homogeneous film on a microscopic scale. Neutron scattering with polarization analysis can discriminate the longitudinal and transverse components of the magnetization. In the experiment four different cross sections can be distinguished, namely the nonspin flip (NSF): $R_{++}$and $\left.R_{--}\right)$and spin flip (SF) channels $\left(R_{+-}\right.$and $\left.R_{-+}\right)$. Here + and signs are used to distinguish the intensity contributions $R$ representing a polarization component $\|$ or anti-\| to the guiding field, respectively. $R_{++/--}$contains the sum/difference between the nuclear and magnetic scattering, whereas the SF signal contains only the magnetic information. A magnetization rotation is identified by a significant increase in the specular SF reflectivities, which corresponds to the formation of in-plane magnetization components developing perpendicular to the guiding field $H_{a}$, applied collinear to $H_{\mathrm{FC}}$. On the other hand, magnetic reversal by domain nucleation and propagation does not provide enhanced SF specular intensities, as long as the domain sizes are smaller than the lateral projection of the neutron coherence length $l_{\|}$(but can produce off-specular signal in the SF channels), because the local magnetization $M$ is always collinear to $H_{a}$. In the specular scattering geometry, normal wave vector transfers $q_{\perp}=(2 \pi / \lambda)\left[\sin \left(\alpha_{i}\right)+\sin \left(\alpha_{f}\right)\right]$ are probed while off-specular scattering contributions along the in-plane momentum transfer vector $q_{\|}=(2 \pi / \lambda)\left[\cos \left(\alpha_{f}\right)-\cos \left(\alpha_{i}\right)\right]$ arise, when the inplane translational symmetry is broken by interface waviness (roughness) or by magnetic domains on a length scale shorter than $l_{\|}$along $q_{\| \cdot}{ }^{11}$

In this letter, we report on the investigation of a multidomain FM by simultaneously analyzing transversal as well 

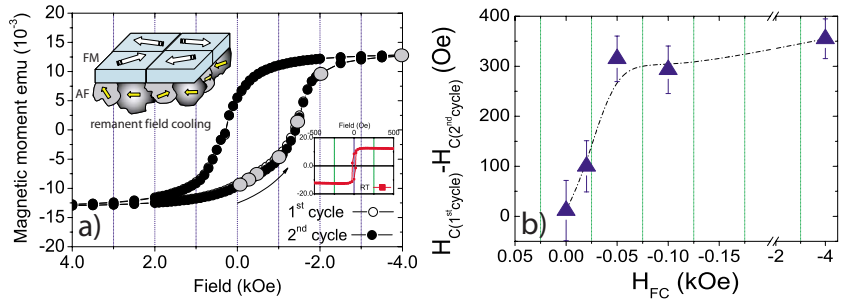

FIG. 1. (Color online) a) SQUID hysteresis loops of $[\mathrm{Co} / \mathrm{CoO} / \mathrm{Au}]_{16} \mathrm{ML}$ during the first (solid symbol) and second field cycles (open symbol) for $H_{\mathrm{FC}}=0$ Oe. The loops correspond to a remanent field cooling state after positive saturation at RT and the arrow along the curve indicates the field sweeping direction. The inset shows the hysteresis loop at RT. The schematic represents the state of the FM-AF layer magnetization after being field cooled. The gray dots are the points of field along the curve used in neutron measurements. b) Degree of training estimated from the $H_{C}$ values (following the SQUID hysteresis loops) during the first and second field cycles for different $H_{\mathrm{FC}}$ after positive saturation at RT. The lines are a guide to the eye.

as longitudinal components of magnetization using the depth sensitive neutron scattering. We show that the suppression of training is unambiguous and is rooted into the micromagnetic state of the interface. Understanding this suppression unravels the basic origin of training and its relation with uniaxial anisotropy of the AF layer.

We have investigated multilayers of the composition $\mathrm{SiO}_{2} /[\mathrm{Co}(9.0 \mathrm{~nm}) / \mathrm{CoO}(7.0 \mathrm{~nm}) / \mathrm{Au}(25.0 \mathrm{~nm})]_{16}$ with different field-cooling options. Details on similar sample growth and sample characterization (structural and magnetic) has been published earlier. ${ }^{3}$ The neutron scattering experiments were performed at the polarized neutron reflectometer with polarization analysis ${ }^{12}$ TREFF at the FRM-II for a wavelength of $4.73 \AA$. In specular geometry the angle of incidence $\alpha_{i}$ equals the final angle $\alpha_{f}$, and $\lambda$ is the wavelength from the monochromator. All measurements have been done after the sample was cooled to $10 \mathrm{~K}$ from RT by a continuous flow cryostat in the presence/absence of a cooling field provided by an electromagnet.

SQUID hysteresis loops of the multilayer during the first and second field cycles for $H_{\mathrm{FC}}=0$ Oe are shown in Fig. 1(a). For $H_{\mathrm{FC}}=0$ Oe (black curve), a shift in the hysteresis loop $\left(H_{E B} \sim-560 \mathrm{Oe}\right)$ is observed after cooling the system in the positive remanent state $\left(+m_{R}\right)$ of the FM. ${ }^{13}$ As we start our field sweep also from positive saturation, the state of the system during its first half of first field cycle is not trained while the loop during second cycle should have been trained, which is obviously not evident here. ${ }^{5}$

The dependence of training on cooling field signifies the role of FM magnetization and its coupling with the AFmagnetization. An increase in $H_{C}$ (half width of the hysteresis loop) has been reported by Paul et al. ${ }^{5}$ Figure 1(b) shows an increasing degree of training (defined as a difference between the $H_{C}$ values for the first and second field cycles) with increasing $H_{\mathrm{FC}}$. Note the loss of macroscopic training for remanent field cooling $\left(H_{\mathrm{FC}}=0 \mathrm{Oe}\right)$. With increase in $H_{\mathrm{FC}}$, more and more $\mathrm{FM}$ domains (aligned at RT) are coupled with a number of AF moments, which eventually increases the strength of the coupling. The degree of training, therefore, attains saturation (along with the saturation of $H_{C}$ ) when the FM moments are saturated at RT before cooling. One may recall that the coercivity enhancement can be attributed to rotatable AF magnetization that are largely ferromagnetically aligned.
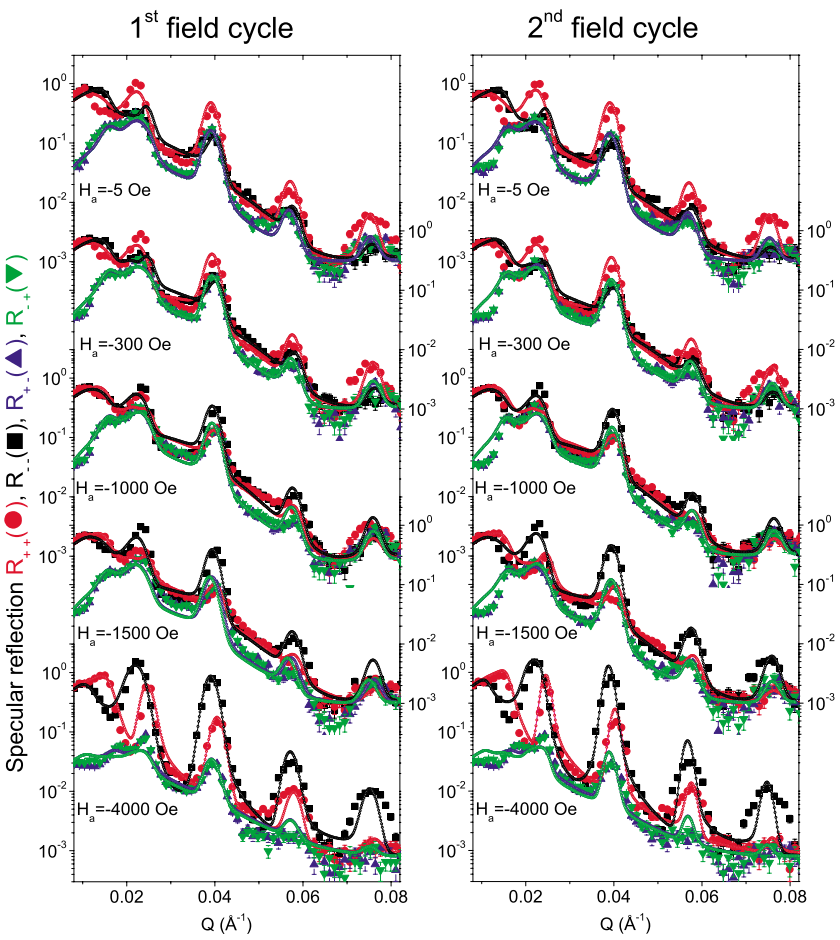

FIG. 2. (Color online) Specular reflectivity patterns for the NSF $\left[R_{++}\right.$(red) and $R_{--}$(black) $]$and SF $\left[R_{-+}\right.$(green) and $R_{+-}$(blue) $]$channels measured from $\mathrm{Co} / \mathrm{CoO} / \mathrm{Au} \mathrm{ML}$ at different $H_{a}$ as indicated after positive saturation. Solid symbols are data and open circles are fits to a model.

The specular reflectivity patterns (closed circle) are shown in Fig. 2 for $H_{\mathrm{FC}}=0$ Oe [taken at seven different $H_{a}$ values along the decreasing branch of the hysteresis loop indicated by closed gray circles in Fig. 1(a)] together with their least-square fits (open circle) for two consecutive cycles of field. ${ }^{6,10}$ The four peaks of the multilayer (ML) in the NSF channels are the respective order of Bragg reflections of the ML. $R_{++}$dominates over $R_{--}$for all fields before the reversal. This is related to a net magnetization, which is collinear to $H_{a}$ during the first and second field cycles.

Figure 3(a) shows a plot of the negligible difference in spectra for the two cycles for two different fields of measurements. The angular variation $\left(\phi_{A}\right)$ of the net magnetization, as deduced from the fits to the specular reflectivity patterns for the first and second field cycles is shown in Fig. 3(b). It is clearly evident that there exists no training for the system and the reversal mechanism is predominantly via uniform rotation of magnetization as is also expected from the typical loop shapes from SQUID.
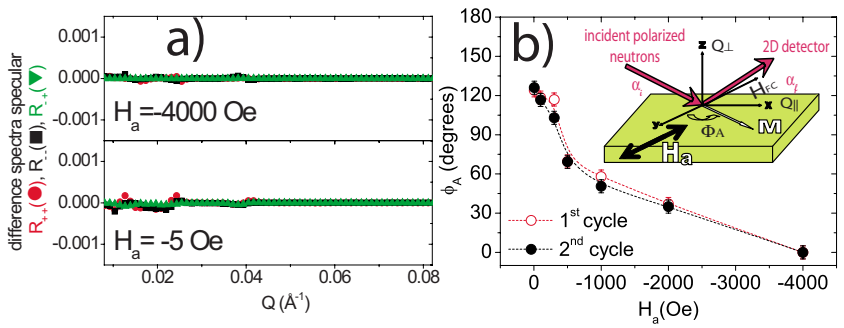

FIG. 3. (Color online) a) Plot of the difference in measured spectra for different cycles at different fields. b) Variation of the angle $\phi_{A}$ as a function of $H_{a}$. The dashed lines are guides to the eye. The schematic shows the scattering geometry. 
1st field cycle
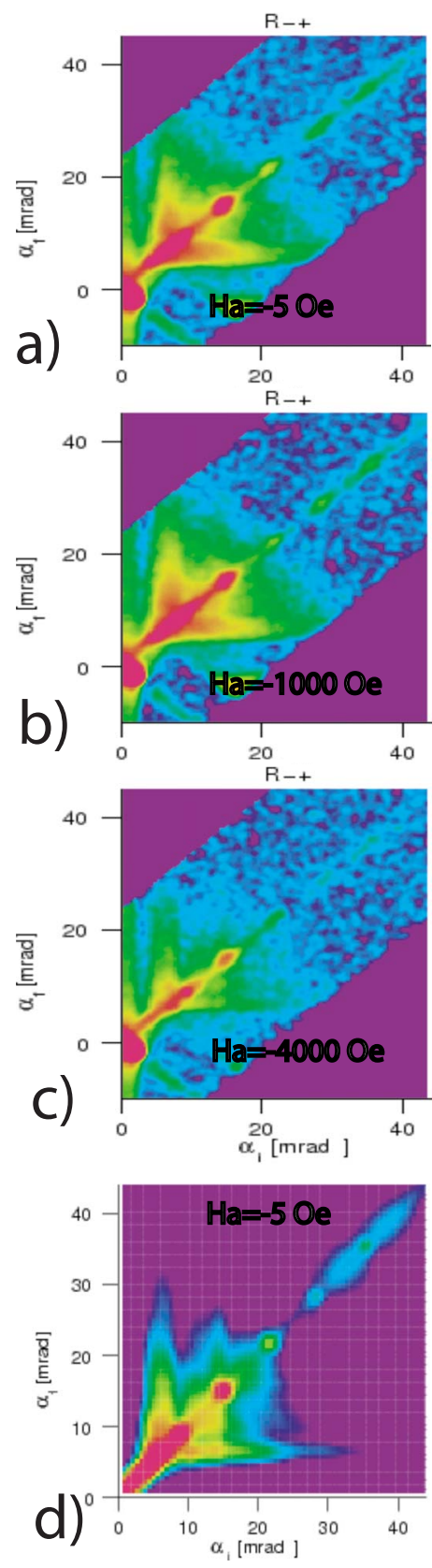

2nd field cycle
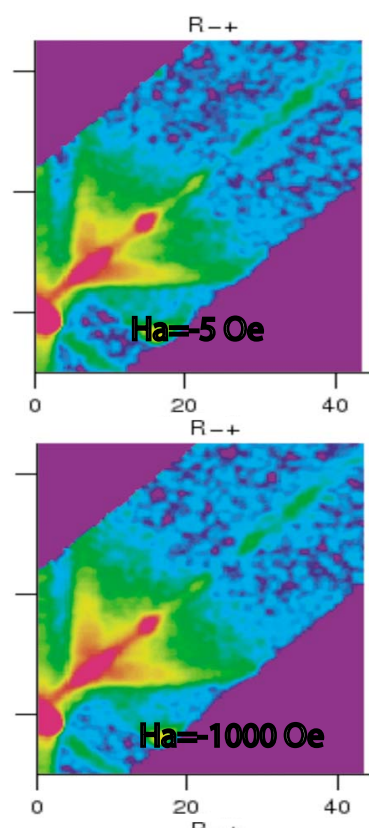

$\mathrm{R}-+$
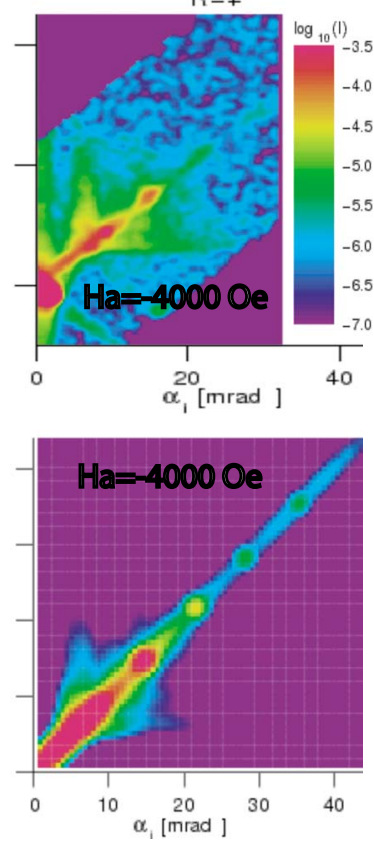

FIG. 4. (Color online) SF intensity maps $\left[R_{-+}\right]$from $\mathrm{Co} / \mathrm{CoO} / \mathrm{Au}$ ML measured at different $H_{a}[(\mathrm{a})-(\mathrm{c})]$ after positive saturation. The color bar encodes the scattered intensity on a logarithmic scale. The simulated SF intensity maps within DWBA (d) are also shown.

The off-specular and specular SF scattering signals shown in Fig. 4, can be attributed to the presence of associated buried magnetic domains on a length scale smaller than $l_{\|}$. These flying-duck like intensity maps (concentrated near the total reflection plateau) are simulated within the supermatrix formalism under the distorted wave born approximation (DWBA), considering the magnetization to fluctuate randomly $\left(\Delta \phi \approx 30^{\circ}\right)$ from domain to domain around the mean angle $\phi_{A} \cdot{ }^{11}$ Vertically uncorrelated domains vary from $\approx 1 \mu \mathrm{m}$ (at remanence) to $\approx 10 \mu \mathrm{m}$ (at saturation). Interesting to note that the intensity maps are very similar irrespective of the two field cycles-indicating again, the untrained state of magnetization along $q_{\perp}$, as well as along $q_{\|}$. It may also be noted that similar simulation of the SF maps at RT gives domain size of $\approx 5 \mu \mathrm{m}$ at remanence and $\approx 25 \mu \mathrm{m}$ at saturation. Locked-in AF state splinters into a collage of domains ${ }^{14}$ from that at RT and when cooled to $10 \mathrm{~K}$.

The direction of the unidirectional anisotropy in the AF and the anisotropy direction of the AF holds the key to the training effect. If the FM-AF coupling is not sufficiently strong, a local unidirectional anisotropy may form in the AF close to the interface that is along an anisotropy direction of the AF, but not necessarily along the FM magnetization direction and this can suppress the training. Within the Fulcomer-Charap model,${ }^{15}$ as the energy barrier is lowered, a larger fraction of the grains, in localized clusters, behave nonhysteretically (rotate back to initial position). It has been conjectured that exchange bias can involve aspects of glassy behavior when cooled down below its transition temperature. ${ }^{16}$ These moments, depending upon the uniaxial anisotropy of the diluted system, can therefore behave either as uniaxial Ising spin (for well defined uniaxial anisotropic layer) or as isotropic Heisenberg spins (for poorly defined uniaxial anisotropic layer). It is well known that magnetic hysteresis can be absent in isotropic Heisenberg system whereas Dzyaloshinsky-Moriya interaction and uniaxial anisotropy effects lead to irreversibility. ${ }^{17}$ This irreversibility, for uniaxially anisotropic layer (causing an increase in $H_{C}$ ), can therefore be identified as the basic origin of the training effect. Thus for relatively isotropic AF, training can be suppressed.

In conclusion, we argue that the confirmation of suppression of training on a microscopic scenario, has not only paved a significant step in understanding of the origin of training but also opened up a new avenue for technological exploitation of untrained remanent exchange bias. A metastable state that can be realized upon lowering the AF energy barrier due to poorly defined uniaxial anisotropy (in absence of cooling field) in a direction away from the FM magnetization holds the key.

${ }^{1}$ W. H. Meiklejohn and C. P. Bean, Phys. Rev. 102, 1413 (1956).

${ }^{2}$ G. Scholten, K. D. Usadel, and U. Nowak, Phys. Rev. B 71, 064413 (2005).

${ }^{3}$ A. Paul, E. Kentzinger, U. Rücker, and Th. Brückel, Phys. Rev. B 74, 054424 (2006).

${ }^{4}$ P. Miltènyi, M. Gierlings, J. Keller, B. Beschoten, G. Güntherodt, U. Nowak, and K. D. Usadel, Phys. Rev. Lett. 84, 4224 (2000).

${ }^{5}$ A. Paul, C. M. Schneider, and J. Stahn, Phys. Rev. B 76, 184424 (2007).

${ }^{6}$ A. Paul, E. Kentzinger, U. Rücker, and Th. Brückel, Phys. Rev. B 73, 092410 (2006).

${ }^{7}$ A. Paul, D. Bürgler, M. Luysberg, and P. Grünberg, Europhys. Lett. 68, 233 (2004).

${ }^{8}$ S. Brems, K. Temst, and C. V. Haesendonck, Phys. Rev. Lett. 99, 067201 (2007).

${ }^{9}$ A. Paul and A. Teichert (unpublished).

${ }^{10}$ A. Paul, E. Kentzinger, U. Rücker, D. Bürgler, and P. Grünberg, Phys. Rev. B 70, 224410 (2004); A. Paul, E. Kentzinger, U. Rücker, D. Bürgler, and Th. Brückel, ibid. 73, 094441 (2006).

${ }^{11}$ B. P. Toperverg, in Polarized Neutron Scattering (Forschungszentrum Jülich, Jülich, Germany, 2002), Vol. 12, p. 247.

${ }^{12}$ A. Paul, M. Buchmeier, D. Bürgler, U. Rücker, and C. Schneider, Phys. Rev. B 77, 184409 (2008).

${ }^{13}$ P. Miltényi M. Gierlings, M. Bamming, G. Günthererodt, J. Nogués, M. Gruyters, C. Leighton, and I. K. Schuller, Appl. Phys. Lett. 75, 2304 (1999).

${ }^{14}$ U. Welp, S. G. E. te Velthuis, G. P. Felcher, T. Gredig, and E. D. Dahlberg, J. Appl. Phys. 93, 7726 (2003).

${ }^{15}$ E. Fulcomer and S. H. Charap, J. Appl. Phys. 43, 4190 (1972).

${ }^{16}$ M. Gruyters, Europhys. Lett. 77, 57006 (2007).

${ }^{17}$ C. M. Soukoulis, G. S. Grest, and K. Levin, Phys. Rev. Lett. 50, 80 (1983). 